\title{
Cooperative Spectrum Sensing for Cognitive Radio Networks with Limited Reporting
}

\author{
Jaewoo So \\ Department of Electronic Engineering, Sogang University \\ Seoul, Republic of Korea \\ [e-mail: jwso@sogang.ac.kr] \\ *Corresponding author: Jaewoo So
}

Received February 10, 2015; revised May 2, 2015; accepted June 30, 2015;

published August 31, 2015

\begin{abstract}
Cooperative spectrum sensing increases the detection performance in a cognitive radio network, based on the number of sensing nodes. However, as the number of sensing nodes increases, the reporting overhead linearly increases. This paper proposes two kinds of cooperative spectrum sensing with limited reporting in a centralized cognitive radio network, a soft combination with threshold-based reporting (SC-TR) and a soft combination with contention-based reporting (SC-CR). In the proposed SC-TR scheme, each sensing node reports its sensing result to the fusion center through its own reporting channel only if the observed energy value is higher than a decision threshold. In the proposed SC-CR scheme, sensing nodes compete to report their sensing results via shared reporting channels. The simulation results show that the proposed schemes significantly reduce the reporting overhead without sacrificing the detection performance too much.
\end{abstract}

Keywords: Cooperative spectrum sensing, limited reporting, cognitive radio network, soft combination, detection probability

This research was supported by the Ministry of Science, ICT and Future Planning (MSIP), Korea, under Grant IITP-2015-H8601-15-1006 of the Convergence Information Technology Research Center (C-ITRC) program supervised by the Institute for Information and Communications Technology Promotion (IITP). It was also supported by the Sogang University Research Grant (No. 201010012). 


\section{Introduction}

Cognitive Radio (CR) enables the efficient use of limited spectrum by allowing secondary users (SUs) to access the licensed frequency bands of primary users (PUs). Spectrum sensing is a key element required to allow SUs to use vacant frequency bands in a CR network. Many signal detection techniques such as energy detection, matched filtering, and cyclostationary feature detection can be used to enhance detection performance in spectrum sensing [1]. However, in practice, many factors, such as multipath fading, shadowing, and the hidden PU problem, may significantly affect detection performance. To solve this challenging problem, cooperative spectrum sensing has been proposed [2]. In cooperative spectrum sensing, each sensing node reports its sensing result to a fusion center (FC). The FC determines the presence of the PU by combining multiple independent sensing results from sensing nodes. A soft combination (SC) based on the Neyman-Pearson criterion can maximize the detection probability for a given false alarm probability [3]. Some researchers have increased the spectrum sensing performance of the energy detector by adjusting parameters such as decision thresholds, sensing frequency, and the number of sensing operations [4]-[6]. However, because the detection performance of the cooperative spectrum sensing increases according to the number of sensing results reported from sensing nodes, it is important to reduce the reporting overhead. The authors of [7], [8] elaborated on the performance of cooperative spectrum sensing while taking the reporting overhead into consideration. To reduce the reporting overhead, cooperative spectrum sensing with quantized sensing observation has been considered in some earlier works [9]-[11]. In [9], [10], the authors formulated a limited reporting scheme that uses one-bit or two-bit quantized information for cooperative spectrum sensing. In [11], SU assignment and sensing observation quantization schemes were proposed in a CR network operating in multiple primary bands. However, the previous studies of [7]-[11] didn't take the reduction of the reporting overheads into consideration. An alternative approach to reduce the reporting overhead is to use difference levels of thresholds in each sensing node. Each sensing node performs local spectrum sensing and decides the presence of a PU by using a different detection threshold. Each sensing node reports its local decision to the FC and then the FC finally decides the presence of a PU by using hard combination (HC) methods (e.g., OR-rule or AND-rule) [12]-[14]. However, the SC method shows better sensing performance than HC methods at the cost of increased bandwidth of reporting channels [9], [15], [16]. In cellular networks with multiuser diversity, various approaches have been studied to reduce the amount of the channel state information fed back by users [17]-[20]. One approach is to quantize the observed channel state [17], [18]. An alternative approach is to allow users to report only if their channel state exceeds a threshold [19], [20]. In cellular networks with multiuser diversity, a base station finds one user who has the best channel condition among multiple users, for every frame. Contrastively, in the CR networks with cooperative spectrum sensing, an FC combines multiple sensing information reported from sensing nodes. Hence, previous multiuser diversity schemes with limited feedback in cellular networks cannot be directly applied to the CR networks.

The paper proposes two cooperative spectrum sensing schemes with dedicated or shared reporting channels. The proposed schemes allow sensing nodes to report their sensing results only if their observed energy is greater than a decision threshold and therefore the proposed schemes result in the reduction of the number of reporting transmissions by sensing nodes. The contribution of this paper is twofold. First, this paper proposes two SC based cooperative 
spectrum sensing schemes, an SC with threshold-based reporting (SC-TR) scheme and an SC with contention-based reporting (SC-CR) scheme. To the best of our knowledge, few studies have reduced the reporting overhead in SC based cooperative CR networks while most previous work has focused on HC based cooperative CR networks. To reduce the reporting overhead, a threshold-based reporting technique proposed for use in cellular networks with multiuser diversity is applied to the proposed schemes and the conventional HC scheme. However, the proposed SC-TR scheme and the HC scheme with threshold-based reporting differ in terms of the combining rule at the FC. The SC based fusion rule can increase the diversity gain by using weighted combining factors. Moreover, the proposed SC-CR scheme can increase the throughput of SUs by using the fixed number of shared reporting channels when there are many SUs. Second, this paper develops an analytical model to evaluate the performance of cooperative spectrum sensing schemes from two perspectives, that of the PU and that of the SU. From the PU's perspective, this paper analyzes the detection probability, the false alarm probability, and the reporting overhead of the cooperative spectrum sensing schemes. From the SU's perspective, this paper analyzes the throughput and the power consumption of SUs. The findings reported in this paper will help operators to find the best cooperative spectrum sensing scheme by comparing the performance of four cooperative spectrum sensing schemes.

The remainder of this paper is organized as follows: Section 2 describes the system model. Section 3 introduces two traditional cooperative spectrum sensing schemes based on the HC and SC method, and also proposes two SC based cooperative spectrum sensing schemes with limited reporting. Section 4 analyzes the sensing performance of four cooperative spectrum sensing schemes in terms of the detection probability, the false alarm probability, and the reporting overhead. Section 5 analyzes the throughput and the power consumption from the SU's perspective. Section 6 presents numerical and simulation results and finally Section 7 concludes the paper.

\section{System Model}

We consider a centralized CR network with $K$ SUs which cooperatively detect the presence of the PU by sending their sensing results to the CR base station, which acts as an FC, as shown Fig. 1.

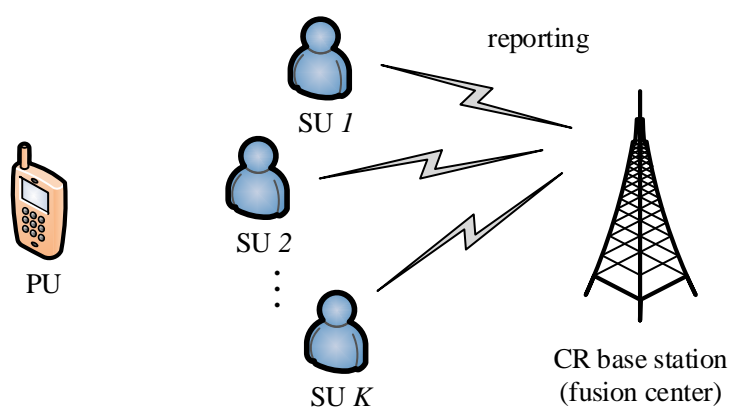

Fig. 1. Cooperative spectrum sensing in a CR network

As shown Fig. 2, each frame of a CR network is divided into sensing duration, reporting duration, and data transmission duration. Let the frame time, sensing time, reporting time, and data transmission time be respectively denoted by $T_{f}, T_{s}, T_{r}$, and $T_{d}$, where $T_{f}=T_{s}+T_{r}+T_{d}$. In 
the sensing duration, each SU $k$ performs spectrum sensing during the sensing time. In the reporting duration, SUs report their sensing results or their observed energy values to the FC via the reporting channels, where the reporting channel between the SU and the FC is assumed to be free of error. The reporting time can be represented by $T_{r}=L \cdot \tau_{r}$, where $L$ is the number of reporting channels and $\tau_{r}$ is the duration of a time slot. The FC determines the presence of a PU by combining multiple independent sensing results from SUs, where we consider two kinds of combination methods at the FC, an HC method and an SC method. For the HC method, an OR-rule scheme is adopted [22]. For the SC method, a maximum ratio combination (MRC) is adopted [9]. In the data transmission duration, if the FC decides the absence of a PU, one SU selected by the FC transmits data, where the FC is assumed to use a round-robin (RR) scheduling algorithm.

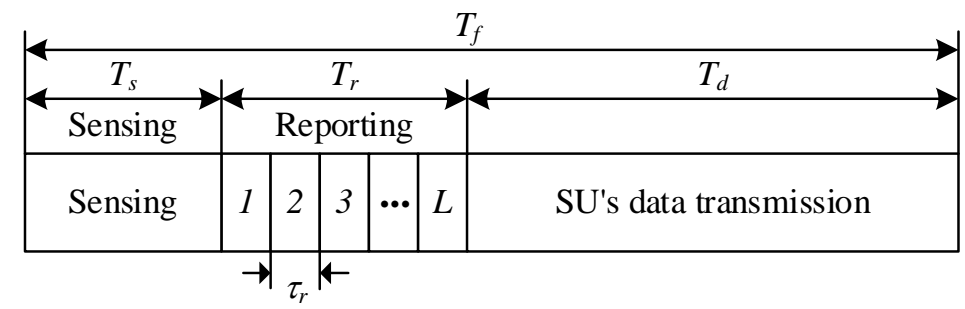

Fig. 2. Frame structure of a CR network

Let the hypotheses corresponding to the absence and presence of the PU signal be denoted by $H_{0}$ and $H_{1}$, respectively. When each SU senses a PU signal during $M$ samples through energy detection, the observed energy value of the $k$ th SU can then be expressed as [21], [22]

$$
Y_{k}= \begin{cases}\sum_{j=1}^{M} n_{k j}^{2}, & H_{0} \\ \sum_{j=1}^{M}\left(s_{k j}+n_{k j}\right)^{2}, & H_{1},\end{cases}
$$

where $s_{k j}$ is the received PU signal and $n_{k j}$ is the white noise at the $j$ th sample of the $k$ th SU, respectively. The noise at each sample is assumed to be Gaussian distributed with zero mean and unit variance, i.e., $n_{k j} \sim \mathcal{N}\left(0, \sigma^{2}\right)$, where $\sigma^{2}=1$. Since different SUs are at different locations, $Y_{k}$ values are assumed to be independent [22]. Let $\gamma_{k}$ represent the received instantaneous SNR of the $k$ th SU within the observation period, defined as $\gamma_{k}=\frac{1}{M} \sum_{j=1}^{M} s_{k j}^{2} / \sigma^{2}$ [9], [22]. We assume $\gamma_{k}$ varies from (observation) period to period. The probability density function and cumulative distribution function of the received SNR of the $k$ th SU are respectively denoted by $f_{\gamma_{k}}\left(\gamma_{k}\right)$ and $F_{\gamma_{k}}\left(\gamma_{k}\right)$.

In the CR network, as the detection probability becomes higher, the PUs are better protected. However, from the perspective of the SU's throughput, as false alarm probability falls, there are more chances the channel can be reused by an SU when it is available, and thus the achievable throughput of SUs becomes accordingly higher. In this light, the CR network has a fundamental tradeoff between the protection of PUs and the achievable throughput of SUs [23]. Consequently, according to the perspective, the objectives of the CR network are as follows: 
- From the PU's perspective, the objective of the CR network is to maximize the detection probability in order to protect PUs while providing the throughput of SUs above a prescribed value; that is, the false alarm probability should be below the prescribed value. The detection performance from the PU's perspective is analyzed in Section 4.

- From the SU's perspective, on the other hand, the objective of the CR network is to maximize the throughput of SUs while providing sufficient protection to the PUs; that is, the detection probability should be greater than a prescribed value. The throughput and the power consumption of SUs from the SU's perspective are analyzed in Section 5.

\section{Cooperative Spectrum Sensing Schemes}

\subsection{OR-rule based Hard Combination}

In the traditional HC-based CR networks, each SU reports its decision result, 0 or 1 , to the FC [12], [14]. However, in this paper, each SU is assumed to report only if the decision result is ' 1 ' to reduce the reporting overhead. In particular, in the HC with different thresholds, each SU $k$ performs local spectrum sensing and decides the presence of the PU on the basis of each threshold, $\mu_{k}$ [12], [14]. Each SU reports one-bit information to the FC via the dedicated reporting channel if the received SNR is greater than each threshold, $\mu_{k}$. Hence, although the number of actually used reporting channels is less than $K$, the number of required reporting channels is given by $L=K$, where $K$ is the number of SUs.

\subsection{Soft Combination with Full Reporting}

In the conventional SC based cooperative spectrum sensing, all SUs report their observed energy values, $Y_{k}$, to the FC via dedicated reporting channels. Hence, the number of reporting channels is given by $L=K$, where $K$ is the number of SUs. The weighted summation at the FC is [15]

$$
Y=\sum_{k=1}^{K} w_{k} Y_{k}
$$

where $w_{k}$, which is the normalized weight coefficient corresponding to the $k$ th SU, is approximated as $w_{k}^{*} \approx \gamma_{k} / \sqrt{\sum_{i=1}^{K} \gamma_{i}^{2}}$ [9], [22]. The FC determines the presence of a PU on the basis of the weighted summation of (2). This operation is called an SC with full reporting (SC-FR).

\subsection{Proposed Soft Combination with Threshold-based Reporting}

In order to reduce the actual reporting overhead, we propose a cooperative spectrum sensing that limits the number of SUs who report their observed energy values; which is called an $S C$-TR. In the SC-TR scheme, each SU is allowed to report its sensing result to the FC through its own reporting channel only if the observed energy value is higher than a decision threshold of $\lambda$ under the hypothesis, $H_{1}$. However, because each SU uses its dedicated reporting channel, the number of required reporting channels is given by $L=K$, where $K$ is the number of SUs. 


\subsection{Proposed Soft Combination with Contention-based Reporting}

Although the SC-TR scheme reduces the actual reporting overhead, each SU requires its own dedicated control channel to report the sensing result to the FC. To prevent a shortage of reporting channels due to the increase in the number of SUs, we propose another cooperative spectrum sensing, which is called an SC-CR, where the reporting channels are shared by all the SUs.

Fig. 3 shows an example of a frame structure of the proposed SC-CR scheme, where the number of reporting channels is fixed to $L=\beta$ regardless of the number of SUs. SUs share $\beta$ reporting channels and compete to send their sensing results through random access from the first reporting channel to the $\beta$ th reporting channel if their observed energy values are greater than a threshold, $\lambda$. SUs randomly access each reporting channel with an access probability, $p$. We assume that the FC can capture the strongest signal from the multiple received signals at each reporting channel. Under a Rayleigh channel, the probability of a signal being captured is given by $i / 2^{i-1}$, where $i$ is the number of received signals [24].

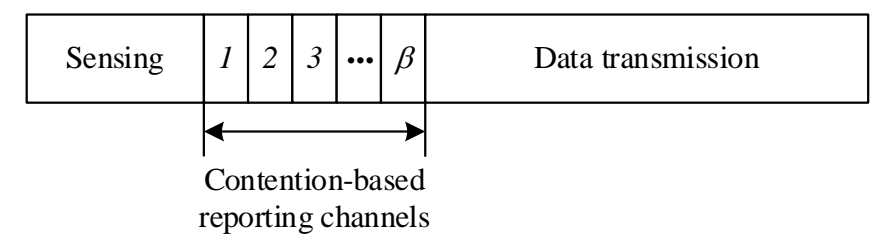

Fig. 3. A frame structure of the SC-CR scheme

Because the SC-CR scheme requires SUs to report their sensing information via contentions, there are pros and cons. The main advantage of the SC-CR scheme lies in the use of a fixed number of reporting channels regardless of the number of SUs. The SC-CR scheme hence can increase the throughput of SUs owing to the fixed number of reporting channels when there are many SUs. Generally, the number of reporting channels linearly increases with the number of SUs and excessive reporting overhead deteriorates the throughput of SUs due to a shortage of available data transmission time [8]. If an FC limits the number of SUs that report their sensing results, the FC will randomly select SUs because it does not know which user has the high observed energy value due to the fluctuation of the channel. Hence, the limitation of the number of SUs may decrease the multiuser diversity gain at the FC. The proposed SC-CR scheme enables the FC to receive sensing results with higher observed energy values via contention based reporting channels by controlling the threshold according to the number of SUs. Hence, the SC-CR scheme increases the multiuser diversity gain at the FC as the number of SUs increases. However, the SC-CR scheme has the following weaknesses: First, the SC-CR scheme deteriorates the performance of the CR networks when the number of SUs is low because of collisions due to the contention based reporting channels. Second, the SC-CR scheme increases the power consumption of SUs because SUs may report their sensing results several times during the reporting time. However, the average power consumption of an SU decreases as the number of SUs increases because the FC controls the reporting load according to the number of SUs. Third, the FC should find the optimal parameters, $\lambda$ and $p$, and inform them to SUs whenever the number of SUs changes. Hence, the SC-CR scheme should be applied to a CR network with many SUs. 


\section{Sensing Performance of the Cooperative Spectrum Sensing Schemes}

\subsection{OR-rule based Hard Combination}

In the HC scheme, because $Y_{k}$ approximates the Gaussian distribution for a large $M$, the probabilities of false alarm and detection of the $k$ th SU are [12], [22]

$$
p_{f, k}=Q\left(\frac{\mu_{k}-M}{\sqrt{2 M}}\right), \quad p_{d, k}=Q\left(\frac{\mu_{k}-M\left(1+\bar{\gamma}_{k}\right)}{\sqrt{2 M\left(1+2 \bar{\gamma}_{k}\right)}}\right),
$$

where $\bar{\gamma}_{k}$ is the average received SNR of the $k$ th SU. When the OR-rule at the FC is applied, the average probabilities of false alarm and detection in the cooperative spectrum sensing network with $K$ SUs are given by

$$
\bar{P}_{F}^{\mathrm{OR}}=1-\prod_{k=1}^{K}\left(1-p_{f, k}\right), \quad \bar{P}_{D}^{\mathrm{OR}}=1-\prod_{k=1}^{K}\left(1-p_{d, k}\right) .
$$

Under the constraint that a false alarm probability is less than a target value, $P_{F}$, the threshold vector, $\boldsymbol{\mu}=\left(\mu_{1}, \mu_{2}, \cdots, \mu_{K}\right)$, is optimally selected to maximize the average detection probability, $\bar{P}_{D}^{\mathrm{OR}}$. The heuristic algorithm to find optimal thresholds was introduced in [14].

Because an SU reports one-bit information only if the observed energy value is greater than its threshold, the number of reporting channels that are actually used by SUs is given by

$$
\bar{N}_{c h}^{\mathrm{OR}}=\sum_{k=1}^{K} p_{d, k}
$$

\subsection{Soft Combination with Full Reporting}

In the SC based fusion rule, given a false alarm probability, $P_{F}$, the detection probability, $P_{D}$, can be expressed as [15], [22]

$$
P_{D}\left(\gamma_{K}\right)=Q\left(\frac{Q^{-1}\left(P_{F}\right) \sqrt{\sum_{j=1}^{K} w_{j}^{2}}-\sqrt{\frac{M}{2}} \sum_{j=1}^{K} w_{j} \gamma_{j}}{\sqrt{\sum_{j=1}^{K} w_{j}^{2}\left(1+2 \gamma_{j}\right)}}\right),
$$

where $Q(x)=\int_{x}^{\infty} \frac{1}{\sqrt{2 \pi}} e^{-t^{2} / 2} d t$ and $\gamma_{K}$ is the vector of the received SNRs at SUs, $\gamma_{K}=\left(\gamma_{1}\right.$, $\left.\gamma_{2}, \cdots, \gamma_{K}\right)$. Similarly, given a detection probability, $P_{D}$, the false alarm probability, $P_{F}$, can be expressed as [22] 


$$
P_{F}\left(\gamma_{K}\right)=Q\left(Q^{-1}\left(P_{D}\right) \sqrt{\sum_{j=1}^{K} w_{j}^{2}\left(1+2 \gamma_{j}\right)}+\sqrt{\frac{M}{2}} \sum_{j=1}^{K} w_{j} \gamma_{j}\right) .
$$

Hence, given a false alarm probability, the average detection probability of the SC-FR scheme can then be expressed as

$$
\bar{P}_{D}^{\mathrm{FR}}=\int_{\gamma_{1}} \int_{\gamma_{2}} \cdots \int_{\gamma_{K}} P_{D}\left(\gamma_{K}\right) \prod_{k=1}^{K} f_{\gamma_{k}}\left(\gamma_{k}\right) d \gamma_{1} d \gamma_{2} \cdots d \gamma_{K} .
$$

Similarly, given a detection probability, the average false alarm probability is given by $\bar{P}_{F}^{\mathrm{FR}}=\int_{\gamma_{1}} \int_{\gamma_{2}} \cdots \int_{\gamma_{K}} P_{F}\left(\gamma_{K}\right) \prod_{k=1}^{K} f_{\gamma_{k}}\left(\gamma_{k}\right) d \gamma_{1} d \gamma_{2} \cdots d \gamma_{K}$. Because all the SUs report their sensing results via dedicated reporting channels, the number of reporting channels that are actually used by SUs is equal to the number of SUs, as follows: $\bar{N}_{c h}^{\mathrm{FR}}=K$, where $K$ is the number of SUs.

\subsection{Proposed Soft Combination with Threshold-based Reporting}

Let $\alpha_{k}(\cdot)$ denote the probability that the observed energy value, $Y_{k}$, of the $k$ th SU is above the threshold $\lambda$ under $H_{1}$. From (1), we obtain $Y_{k}=M\left(\gamma_{k}+1\right)$ and therefore the decision threshold can be represented by $\lambda=M\left(\lambda_{t h}+1\right)$. The $\alpha_{k}\left(\lambda_{t h}\right)$ can then be expressed as

$$
\alpha_{k}\left(\lambda_{t h}\right)=\int_{\lambda_{t h}}^{\infty} f_{\gamma_{k}}\left(\gamma_{k}\right) d \gamma_{k}
$$

The probability that there are $n$ SUs who have an observed energy value higher than a threshold is

$$
U(n)=\left(\begin{array}{c}
K \\
n
\end{array}\right) \prod_{k=1}^{n} \alpha_{k}\left(\lambda_{t h}\right) \prod_{k=n+1}^{K}\left(1-\alpha_{k}\left(\lambda_{t h}\right)\right)
$$

When $n$ SUs out of $K$ SUs report their observed energy values to the FC, the weighted summation at the FC is given by

$$
Y=\sum_{i=1}^{n} w_{i} Y_{i}, \quad n \leq K
$$

where $Y_{i}$ is the observed energy value of the $i$ th reported message. Let $\Gamma(n)$ denote the set of the SNRs listed in descending order as follows:

$$
\Gamma(n)=\left\{\left(\gamma_{1}, \gamma_{2}, \cdots, \gamma_{n}, \gamma_{n+1}, \cdots, \gamma_{K}\right) \mid \gamma_{i} \geq \gamma_{i+1}, \gamma_{n}>\lambda_{t h}, \gamma_{n+1} \leq \lambda_{t h}\right\} .
$$


Given a false alarm probability, the average detection probability of the SC-TR scheme can then be expressed as

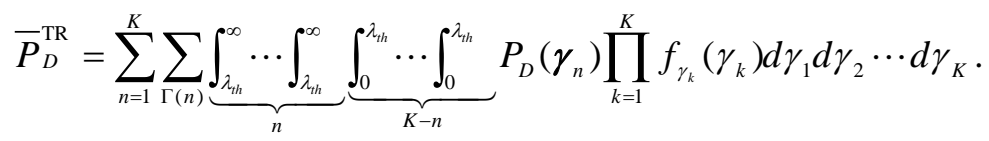

where $\gamma_{n}$, which is the ordered list of the received SNRs of SUs with $\gamma_{k}>\lambda_{t h}$, is given by $\gamma_{n}=\left(\gamma_{1}, \gamma_{2}, \cdots, \gamma_{n}\right)$. The $P_{D}\left(\gamma_{n}\right)$ is obtained from (6). The case in which there is no reporting transmissions from SUs can be neglected if the number of SUs is large enough. Similarly, given the detection probability, the average false alarm probability is obtained from (13) by replacing $P_{D}\left(\gamma_{n}\right)$ with $P_{F}\left(\gamma_{n}\right)$.

Because the number of SUs who can report their sensing results is limited to the SUs who have the observed energy value higher than a threshold, the average number of reporting channels that are actually used by SUs is given by

$$
\bar{N}_{c h}^{\mathrm{TR}}=\sum_{n=1}^{K} n U(n) .
$$

If the received SNRs of SUs are identically distributed, we have $\bar{N}_{c h}^{\mathrm{TR}}=K \alpha_{k}\left(\lambda_{t h}\right)$.

\subsection{Proposed Soft Combination with Contention-based Reporting}

Assuming that there are $n$ SUs whose observed energy values are greater than $\lambda$, let $p_{s}(n)$ be the probability that one SU out of $n$ candidate SUs successfully reports its sensing result at a contention based reporting channel, as follows: $p_{s}(n)=\sum_{i=1}^{n}\left(\begin{array}{l}n \\ i\end{array}\right) p^{i}(1-p)^{n-i} i / 2^{i-1}$, where $p$ is the access probability. Because an SU do not know if the reported signal is succssfully received at the $\mathrm{FC}$, the $\mathrm{SU}$ may report its sensing result several times during the $\beta$ reporting channels and the FC may receive multiple sensing results from the same SU. The probability that there are $m$ different sensing results out of $k$ successfully received sensing results is given by

$$
Q_{s}(k, m \mid n)=\left(\begin{array}{l}
n \\
m
\end{array}\right) \operatorname{suj}(k, m) / \sum_{i=1}^{\min (k, n)}\left(\begin{array}{l}
n \\
i
\end{array}\right) \operatorname{suj}(k, i)
$$

where $\left(\begin{array}{l}n \\ m\end{array}\right) \operatorname{suj}(k, m)$ is the number of cases that $k$ sensing results are reported by $m$ SUs when there are $n$ candidate SUs, and $\operatorname{suj}(k, m)$, which is the number of surjections from an $k$-set to an $m$-set, is given by $\operatorname{suj}(k, m)=\sum_{i=0}^{m-1}(-1)^{i}\left(\begin{array}{c}m \\ i\end{array}\right)(m-i)^{k}$. Consequently, when there are $n$ candidate SUs, the probability that $m$ SUs out of $n$ candidate SUs successfully report their 
observed energy values without collision is given by

$$
V(n, m)=U(n) \sum_{k=m}^{\beta}\left(\begin{array}{l}
\beta \\
k
\end{array}\right) p_{s}(n)^{k}\left(1-p_{s}(n)\right)^{\beta-k} Q_{s}(k, m \mid n), \quad m \leq \min (n, \beta) .
$$

The average detection probability of the SC-CR scheme can then be expressed as

$$
\begin{aligned}
& \bar{P}_{D}^{\mathrm{CR}}(\lambda, p, \beta)=\sum_{n=1}^{K} \sum_{m=1}^{\min (n, \beta)} \sum_{\Gamma(n)} V(n, m)
\end{aligned}
$$

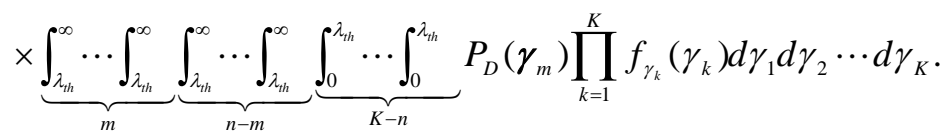

where $\gamma_{m}$ is the vector of the received SNRs of SUs that successfully report their sensing results without collision. $P_{D}\left(\gamma_{m}\right)$ is obtained from (6). For simplicity, we neglect the case in which there is no successful reporting from SUs, i.e, for $n=0$ or $m=0$, the detection probability is assumed to be zero. Similarly, given the detection probability, the average false alarm probability is obtained from (17) by replacing $P_{D}\left(\gamma_{m}\right)$ with $P_{F}\left(\gamma_{m}\right)$.

The probability that at least one SU out of $n$ candidates SUs transmits its sensing result at a reporting channel is $p_{t x}(n)=1-(1-p)^{n}$. The average number of reporting channels that are actually used by SUs can then be expressed

$$
\begin{aligned}
\bar{N}_{c h}^{\mathrm{CR}} & =\sum_{n=1}^{K} U(n) \sum_{j=1}^{\beta} j\left(\begin{array}{c}
\beta \\
j
\end{array}\right) p_{t x}(n)^{j}\left(1-p_{t x}(n)\right)^{\beta-j} \\
& =\beta \sum_{n=1}^{K} U(n)\left(1-(1-p)^{n}\right) .
\end{aligned}
$$

With a fixed number of reporting channels, $\beta$, the parameters of $\lambda$ and $p$ are selected to maximize the average detection probability as follows:

$$
\begin{gathered}
\left(\lambda^{*}, p^{*}\right)=\arg \max _{\lambda, p} \bar{P}_{D}^{\mathrm{CR}}(\lambda, p, \beta) \\
\text { s.t. } \quad 0 \leq p \leq 1, \quad 0 \leq \lambda \leq \lambda_{\max }, \quad \bar{P}_{F} \leq \varepsilon
\end{gathered}
$$

In (19), since the decision threshold $\lambda$ and the access probability $p$ are independent and the detection probability of cooperative spectrum sensing increases with the number of sensing results reported from SUs, we can select the value of $p^{*}$ that maximizes $V(n, m)$. Hence, when there are $n$ SUs whose observed energy values are greater than $\lambda$, the optimization problem to find the optimal access probability is given by 


$$
p^{*}=\arg \max _{p} V(n, m) .
$$

Because the solution of $\partial V(n, m) / \partial p=0$ is identical to $\partial p_{s}(n) / \partial p=0$, the optimal value is given by $p^{*}=2 / n$. However, because the FC cannot precisely estimate the number of candidate SUs for every sensing period, the FC estimates the number of candidate SUs on the basis of the distribution of the received SNR as follows:

$$
\tilde{n}=\sum_{k=1}^{K} \int_{\gamma_{t h}}^{\infty} f_{\gamma_{k}}(\gamma) d \gamma
$$

Consequently, the optimal value of $p$ is estimated by $p^{*} \approx \min (2 / \tilde{n}, 1.0)$. With a given value of $p^{*}$, the optimal value of the threshold is selected to maximize (19) by using the Golden section method or an exhaust search method [25].

\section{Throughput and Power Consumption of Cooperative Spectrum Sensing Schemes}

\subsection{Throughput of Secondary Users}

In the cooperative CR network, there is a tradeoff between the sensing-reporting time and the data transmission time. A longer sensing and reporting time will improve the sensing performance; however, this shortens the allowable data transmission time, which degrades the throughput of SUs. A cooperative fusion method also affects the throughput of SUs because the throughput of SUs depends on the sensing performance. Generally, the SC method shows better sensing performance than the HC methods [15], [16]. Given the sensing time and the average detection probability, the average spectral efficiency of an SU can then be expressed as [23]

$$
\bar{R}=\left(1-\Delta_{s}-L \Delta_{r}\right)\left(r_{0}\left(1-\bar{P}_{F}\right) P\left(H_{0}\right)+r_{1}\left(1-\bar{P}_{D}\right) P\left(H_{1}\right)\right)[\mathrm{bps} / \mathrm{Hz}]
$$

where $\Delta_{s}=T_{s} / T_{f}, \Delta_{r}=\tau_{r} / T_{f}$, and $L$ is the number of reporting channels. Additionally, $r_{0}$ is the average spectral efficiency of an SU when the primary channel is idle and $r_{1}$ is the average spectral efficiency of an SU when the primary channel is busy. From (22), the average throughput of an SU depends on the sensing time, the number of reporting channels, the average false alarm probability, and the average detection probability because the other parameters, $r_{0}, r_{1}, P\left(H_{0}\right)$, and $P\left(H_{1}\right)$, are uncontrollable. To provide sufficient protection to the PUs, the throughput of SUs should be derived under the constraint that the detection probability is greater than a prescribed value.

In the SC-CR scheme, the parameters, $\lambda$ and $p$, are selected to maximize the average spectral efficiency of an SU as follows:

$$
\begin{gathered}
\left(\lambda^{*}, p^{*}\right)=\arg \max _{\lambda, p} \bar{R} \\
\text { s.t. } \quad 0 \leq p \leq 1, \quad 0 \leq \lambda \leq \lambda_{\max }, \quad \bar{P}_{D} \geq \xi,
\end{gathered}
$$


where $\xi$ is the prescribed value to provide protection to the PUs. Since $r_{0}, r_{1}, P\left(H_{0}\right)$, and $P\left(H_{1}\right)$ are uncontrollable, the above optimization problem of (23) can be simplified as $\min _{\lambda, p} \bar{P}_{F}^{\mathrm{CR}}$. The optimal value of $p$ is obtained from (20) and the optimal value of $\lambda$ is selected to minimize the average false alarm probability by using the Golden section method.

\subsection{Power Consumption of Secondary Users}

The power consumption of an SU during the reporting time can be expressed as

$$
P_{c}=P_{\text {proc }}+N_{t} \cdot P_{t}
$$

where $P_{\text {proc }}$ is the processing power, $P_{t}$ is the power consumed to transmit a reporting message, and $N_{t}$ is the number of reporting transmissions during the reporting time. Because an SU mainly consumes power to transmit a message, the power consumption of an SU can be approximately represented by $P_{c} \approx N_{t} \cdot P_{t}$, where $P_{t}$ is assumed to be a constant value in this paper. According to the cooperative spectrum schemes, the average number of reporting transmissions of an SU, $\bar{N}_{t}$, is as follows:

- For the OR-rule based HC scheme, because each SU $k$ reports its sensing result if the received SNR is greater than its threshold, $\mu_{k}$, the average number of reporting transmissions is given by $\bar{N}_{t}^{\mathrm{OR}}=\frac{1}{K} \sum_{k=1}^{K}\left(1-F_{\gamma_{k}}\left(\mu_{k}\right)\right)$.

- $\quad$ For the SC-FR scheme, because each SU reports its sensing result via its own control channel, the average number of reporting transmissions is given by $\bar{N}_{t}^{\mathrm{FR}}=1$.

- For the SC-TR scheme, because each SU reports its sensing result only when the received SNR is greater than the threshold, $\lambda_{t h}$, the average number of reporting transmissions is given by $\bar{N}_{t}^{\mathrm{TR}}=\frac{1}{K} \sum_{k=1}^{K}\left(1-F_{\gamma_{k}}\left(\lambda_{t h}\right)\right)$.

- For the SC-CR scheme, because each SU may transmit its sensing result several times in $\beta$ reporting channels with an access probability of $p$ if the received SNR is greater than the threshold, $\lambda_{\text {th }}$, the average number of reporting transmissions is given by $\bar{N}_{t}^{\mathrm{CR}}=\beta p \frac{1}{K} \sum_{k=1}^{K}\left(1-F_{\gamma_{k}}\left(\lambda_{t h}\right)\right)$. Hence, the power consumption of the SC-CR scheme is higher than that of the SC-TR scheme by $\beta \cdot p$ times.

\section{Numerical and Simulation Results}

\subsection{Simulation Environments}

The performance of the cooperative spectrum sensing is evaluated under a Rayleigh channel environment with $f_{\gamma_{k}}(\gamma)=1 / \bar{\gamma}_{k} \exp \left(-\gamma / \bar{\gamma}_{k}\right)$, where $\gamma$ is the received PU's SNR at the $k$ th SU and $\bar{\gamma}_{k}$ is the average SNR of the $k$ th SU. For simplicity, the average SNRs of SUs are assumed to be uniformly distributed among three values of $-12 \mathrm{~dB},-9 \mathrm{~dB}$, and $-6 \mathrm{~dB}$, respectively. However, the results can be generalized without modification by modeling the distribution of the average SNR. Each SU is assumed to sense a PU signal during $M=25$ samples. 
Because of the tradeoff between the PU's protection and the SU's throughput, we respectively evaluate the performance of four cooperative spectrum sensing schemes from the PU's perspective and from the SU's perspective. First, from the perspective of the PU's protection, we compare the sensing performance of the four cooperative spectrum sensing schemes in Fig. 4 to Fig. 7, with a given value of a false alarm probability, $\varepsilon=0.05$. Here, to calculate the multi-fold integration in (8), (13), and (17), we reduce the multiple integral to an iterated integral (i.e., a series of integrals of one variable) and use a trapezoidal rule for approximating each definite integral. Second, from the SU's perspective, we compare the spectral efficiency and the power consumption of an SU in Fig. 8 and Fig. 9 with the given value of a detection probability, $\xi=0.9$. The parameters used in the simulation are as follows: $P\left(H_{0}\right)=0.8, P\left(H_{1}\right)=0.2, r_{0}=2.6 \mathrm{bps} / \mathrm{Hz}, r_{1}=1.9 \mathrm{bps} / \mathrm{Hz}, \Delta_{s}=0.1, \Delta_{r}=0.01$ in the SC method and $\Delta_{r}=0.005$ in the HC method. In the simulation results of Fig. 4 to Fig. 9, the symbols represent the computer simulation results and the lines represent the analytical results. For the OR-rule based HC scheme, only analytical results are plotted.

\subsection{Sensing Performance from the PU's Perspective}

Fig. 4 shows the average detection probabilities of the four cooperative spectrum sensing schemes. The SC-FR scheme shows the best detection performance at the cost of increased reporting overhead and the OR-rule based HC scheme shows the worst detection performance. For the three SC schemes, the detection probability approaches one as the number of SUs increases. However, for the OR-rule based HC scheme, the detection probability slowly increases as the number of SUs increases because the HC scheme has low sensing performance at the low SNR region. In the SC-TR scheme, as the decision threshold decreases, the detection performance increases with an increase of the reporting overhead. The SC-CR scheme shows the worse detection performance because of collisions due to the shared reporting channels. When the number of SUs is 42, the loss of the average detection performance is about $1.9 \%$ in the SC-TR scheme with $\lambda_{\text {th }}=-7 \mathrm{~dB}$, about $3.3 \%$ in the SC-TR scheme with $\lambda_{\text {th }}=-6 \mathrm{~dB}$, about $9.3 \%$ in the SC-CR scheme, and about $45.2 \%$ in the OR-rule based HC scheme, in comparison with the SC-FR scheme.

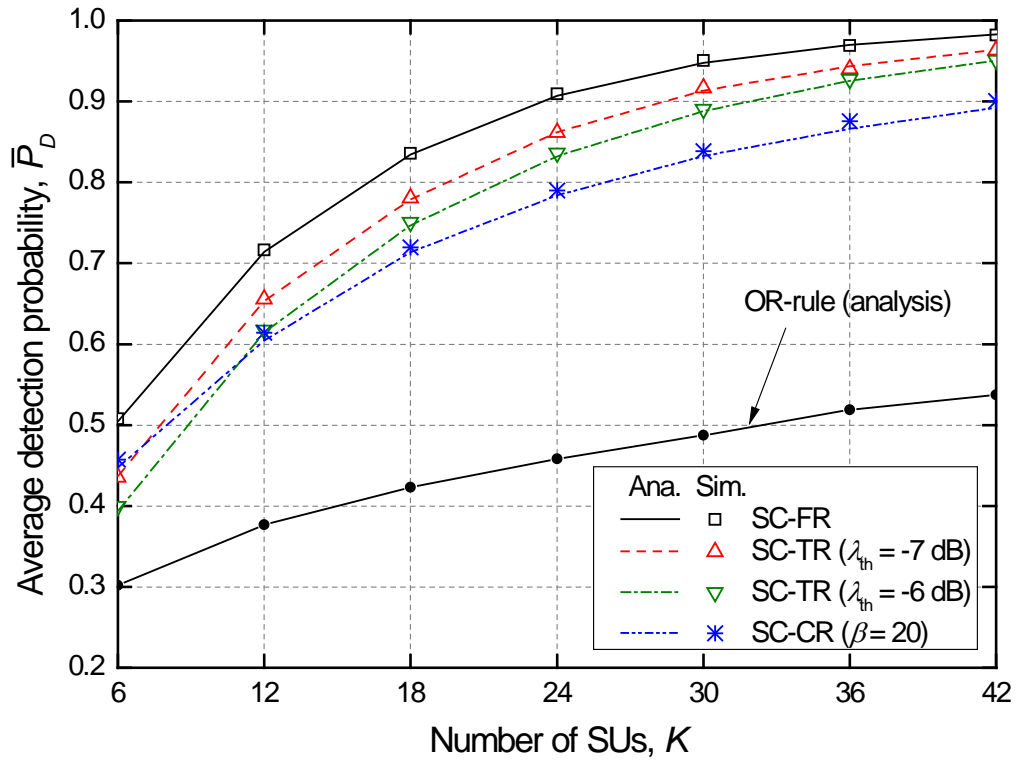

Fig. 4. The average detection probability vs. the number of SUs 
Fig. 5 illustrates the average number of reporting channels that are actually used by SUs. In the conventional SC-FR scheme, the reporting overhead linearly increases with the number of SUs because all SUs report their sensing results via the dedicated reporting channels. In the SC-TR scheme, the number of dedicated reporting channels also increases with the number of SUs but the number of reporting channels that are actually used by SUs can be adjusted by controlling the value of the threshold, $\lambda$. In particular, the SC-CR scheme uses a fixed number of reporting channels regardless of the number of SUs; moreover, the number of reporting channels actually used by SUs is also almost fixed under the value of $\beta$. When the number of SUs is 42 , the average number of actually used reporting channels can be reduced by about $76.7 \%$ in the SC-TR scheme with $\lambda_{\text {th }}=-7 \mathrm{~dB}$, about $82.6 \%$ in the SC-TR scheme with $\lambda_{\text {th }}=-6$ $\mathrm{dB}$, and about $58.3 \%$ in the SC-CR scheme, in comparison with the SC-FR scheme. In particular, for the OR-rule based HC scheme, a very small number of reporting channels are actually used by SUs in order to report one-bit information because, if at least one SU reports one-bit information, the FC decides the presence of a PU.

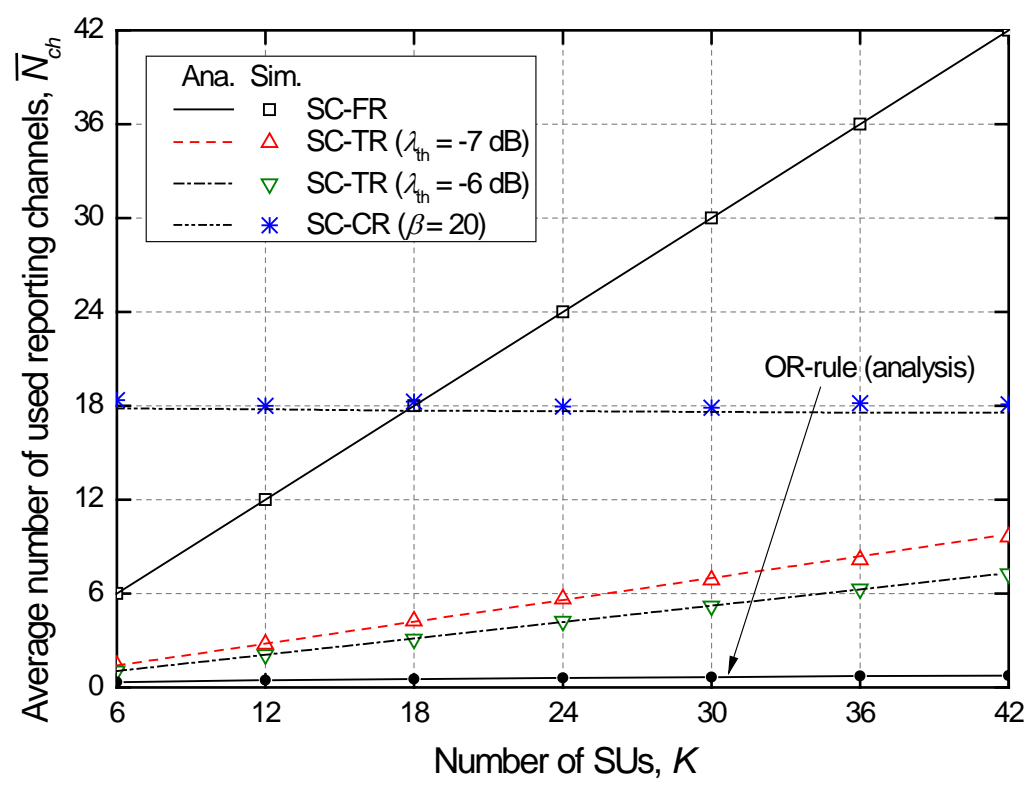

Fig. 5. The average number of used reporting channels vs. the number of SUs

Fig. 6 shows the average detection probability of the SC-CR scheme according to the number of reporting channels. As expected, the detection performance increases as the number of reporting channels increases. For a small number of $\beta$, the detection performance is severely deteriorated because of frequent collisions. For $K=42$, the average detection probability is about $\{0.82,0.86,0.89,0.92\}$ when $\beta=\{10,15,20,30\}$, respectively. 


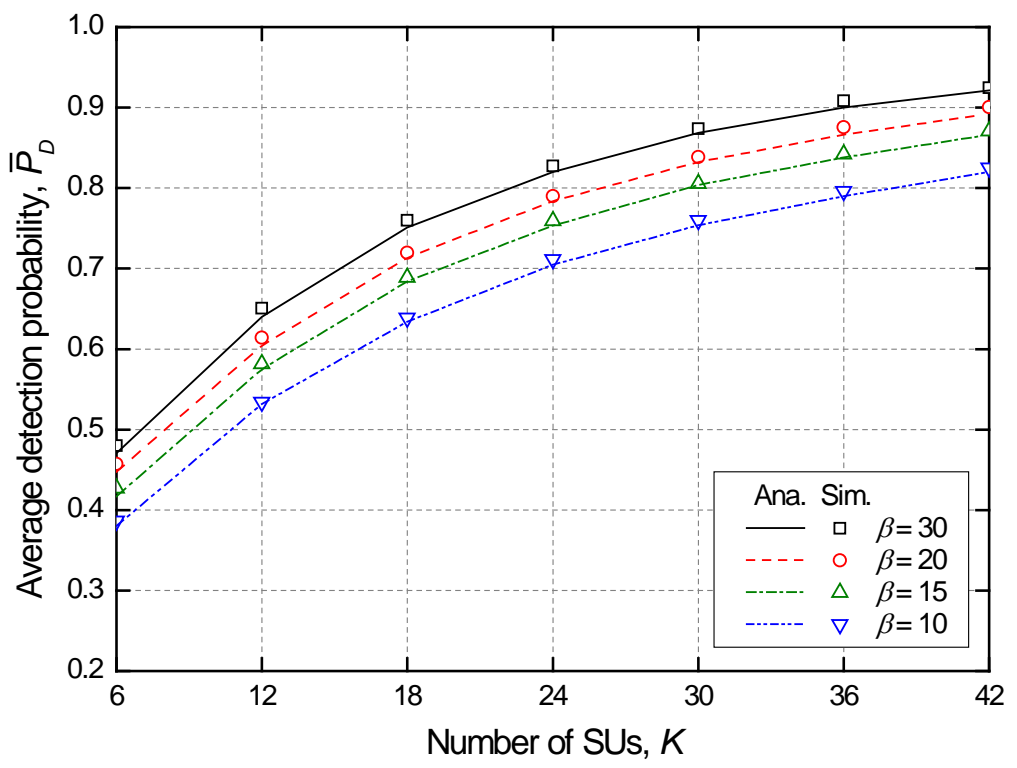

Fig. 6. The average detection probability of the SC-CR scheme

Fig. 7 shows the average detection probability as the average received SNR of SUs increases when the number of SUs is 24, where the average SNRs of all SUs are assumed to be identical. The average detection probability approaches to one as the average received SNR of SUs increase. The OR-rule based HC scheme shows the worst detection performance. In particular, the detection probability of the OR-rule based HC scheme is significantly deteriorated at the low SNR region. The decision threshold of the SC-TR scheme is configured by adding $2 \mathrm{~dB}$ to the average SNR of SUs. When the average SNR is $-8 \mathrm{~dB}$, the loss of the average detection performance is about $9.4 \%$ in the SC-TR scheme, about $17.9 \%$ in the SC-CR scheme, and about $53.9 \%$ in the OR-rule based HC scheme, in comparison with the SC-FR scheme.

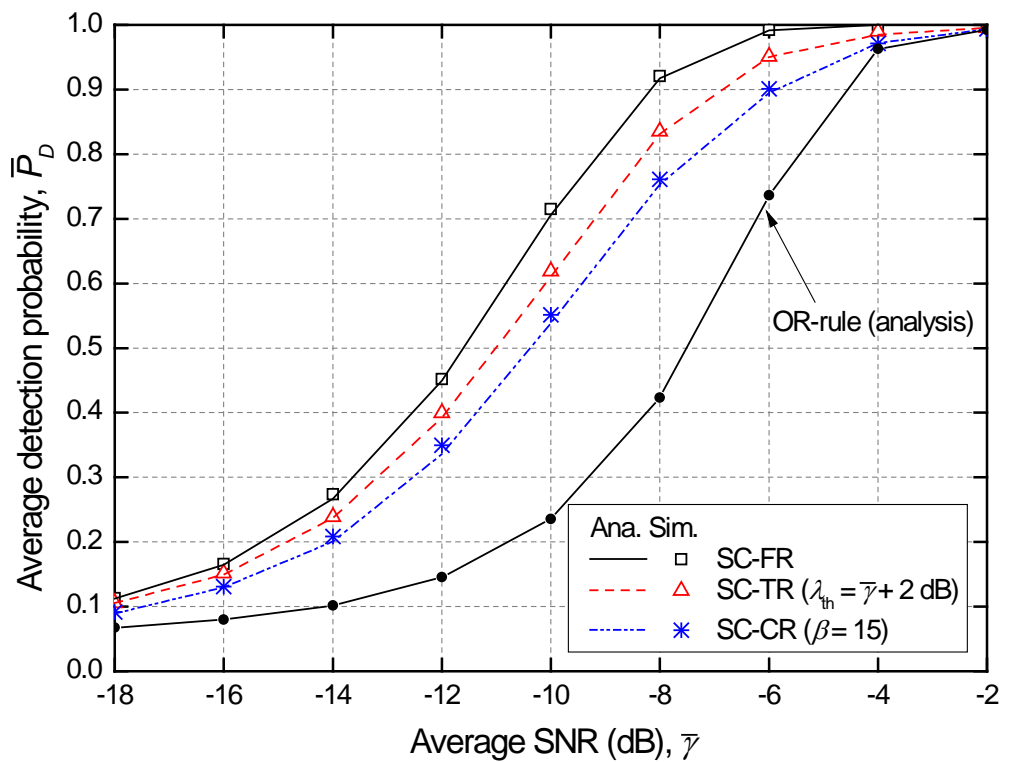

Fig. 7. The average detection probability vs. the average SNR 


\subsection{Throughput and Power Consumption from the SU's Perspective}

Fig. 8 shows the average spectral efficiency of an SU in the four cooperative spectrum sensing schemes, where the parameters of the SC-CR scheme are optimally selected to maximize the throughput of an SU. In the SC-FR and SC-TR schemes, as the number of SUs increases, the average spectral efficiency of an SU increases owing to the increase of the sensing performance but it decreases after a critical number of SUs, e.g., 18 in the SC-FR scheme, because of the excessive reporting overhead. On the other hand, the average spectral efficiency of the SC-CR scheme continuously increases without any decrease owing to the multiuser diversity as the number of SUs increases. However, the SC-CR scheme shows the worst throughput performance when the number of SUs is low. Although the OR-rule based HC scheme uses a small amount of resources to transmit a reporting message, it shows worse throughput performance because of its lower sensing performance.

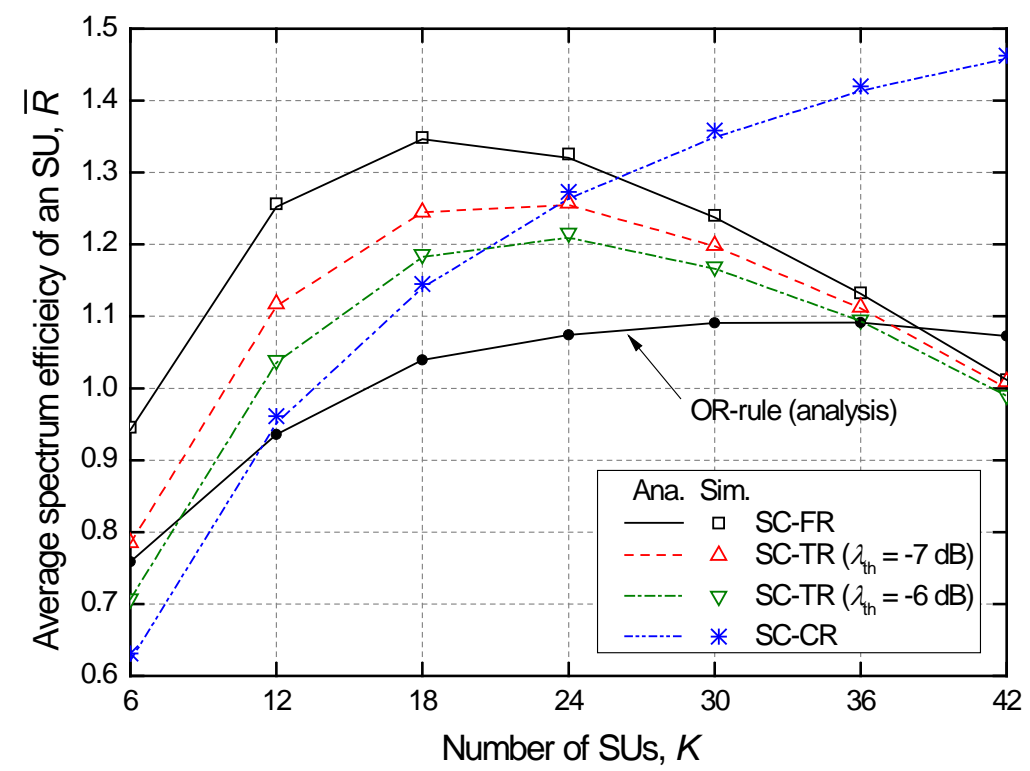

Fig. 8. The average spectral efficiency of an SU vs. the number of SUs

Fig. 9 shows the average number of reporting transmissions of an $\mathrm{SU}$ in the four cooperative spectrum sensing schemes, where the parameters of the SC-CR scheme are optimally selected to maximize the throughput. From (24), the average number of reporting transmissions can be approximately regarded as the amount of power consumption. In the SC-FR scheme, because all SUs report their sensing results during the reporting time, the power consumption of an SU becomes $P_{t}$, where $P_{t}$ is the power consumed to transmit a reporting message. In the SC-TR scheme and the OR-rule based HC scheme, the power consumption of an SU is very low and it is almost constant because an SU transmits its sensing result only when the observed energy value is greater than a threshold. The OR-rule based HC scheme has a higher threshold because, if at least one SU reports one-bit information, the FC decides the presence of a PU. Hence, in the OR-rule based HC scheme, the power consumption of an SU is quite low because of the higher threshold. In the SC-CR scheme, SUs may transmit their reporting messages several times during the reporting time and therefore the power consumption of an SU may be higher. However, because the FC controls the load of reporting transmissions according to the number of SUs, the average power consumption of an 
SU decreases as the number of SUs increases.

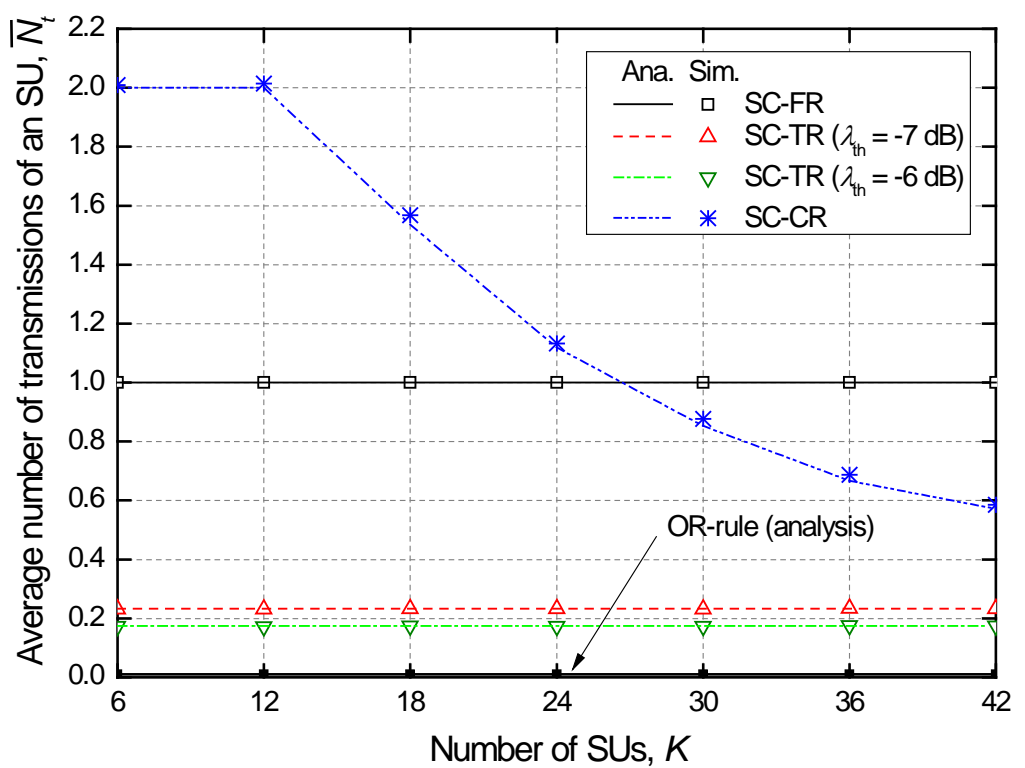

Fig. 9. The average number of reporting transmissions of an SU vs. the number of SUs

\section{Conclusion}

In a centralized cognitive radio network, two cooperative spectrum sensing schemes have been proposed to reduce the reporting overhead; a soft combination with threshold-based reporting (SC-TR) and a soft combination with contention-based reporting (SC-CR). The proposed schemes significantly reduce the reporting overhead with a slight sacrifice in detection performance. When the number of sensing nodes is 42 , the SC-TR scheme reduces the actual reporting overhead by about $76.7 \%$ with an $1.9 \%$ sacrifice in detection performance; and the SC-CR scheme reduces the reporting overhead by about $58.3 \%$ with a $9.3 \%$ sacrifice in detection performance. Additionally, we can control the tradeoff between the reduction of the reporting overhead and the loss of the detection performance by adjusting the decision threshold in the SC-TR scheme or the number of shared reporting channels in the SC-CR scheme. From the perspective of secondary users, we analyzed the throughput and power consumption of an SU. When there are many secondary users, the SC-CR scheme shows higher throughput with an increase of the power consumption than other threshold-based cooperative spectrum sensing schemes.

\section{References}

[1] T. Yücek and H. Arslan, "An survey of spectrum sensing algorithms for cognitive radio applications," IEEE Communications Surveys \& Tutorials, vol. 11, no. 1, pp. 116-130, First quarter 2009. Article (CrossRef Link).

[2] Y.-C. Liang, K.-C. Chen, G. Y. Li, and P. Mahonen, "Cognitive radio networking and communications: an overview," IEEE Transactions on Vehicular Technology, vol. 60, no. 711, pp. 3386-3407, Sep. 2011. Article (CrossRef Link). 
[3] E. C.Y. Peh, Y.-C. Liang, Y. L. Guan, and Y. Zeng, "Cooperative spectrum sensing in cognitive radio networks with weighted decision fusion schemes," IEEE Transactions on Wireless Communications, vol. 9, no. 12, pp. 3838-3847, Dec. 2010. Article (CrossRef Link).

[4] H. Hu, Y. Xu, Z. Liu, N. Li, and H. Zhang, "Optimal strategies for cooperative spectrum sensing in multiple cross-over cognitive radio networks," KSII Transactions on Internet and Information Systems, vol. 6, no. 12, pp. 3016-3080, Dec. 2012. Article (CrossRef Link).

[5] X.-L. Huang, G. Wang, F. Hu, and S. Kumar, "The impact of spectrum sensing frequency and packet-loading scheme on multimedia transmission over cognitive radio networks," IEEE Transactions on Multimedia, vol. 13, no. 4, pp.748-761, Aug. 2011. Article (CrossRef Link).

[6] W. Lee and D.-H. Cho, "Enhanced spectrum sensing scheme in cognitive radio systems with MIMO antennae," IEEE Transactions on Vehicular Technology, vol. 60, no. 3, pp. 1072-1085, Mar. 2011. Article (CrossRef Link).

[7] A. Noel and R. Schober, "Convex sensing-reporting optimization for cooperative spectrum sensing," IEEE Transactions on Wireless Communications, vol. 11, no. 5, pp. 1900-1910, May 2012. Article (CrossRef Link).

[8] Y.-J. Choi, W. Pak, Y. Xin, and S. Rangarajan, "Throughput analysis of cooperative spectrum sensing in Rayleigh-faded cognitive radio systems," IET Communications, vol. 6, no. 9, pp. 1104-1110, Sep. 2012. Article (CrossRef Link).

[9] J. Ma, G. Zhao, and Y. G. Li, "Soft combination and detection for cooperative spectrum sensing in cognitive radio networks," IEEE Transactions on Wireless Communications, vol. 7, no. 11, pp. 4502-4507, Nov. 2008. Article (CrossRef Link).

[10] Z. Wang and W. Zhang, "Exploiting multiuser diversity with 1-bit feedback for spectrum sharing," IEEE Transactions on Wireless Communications, vol. 62, no. 1, pp. 29-40, Jan. 2014. Article (CrossRef Link).

[11] P. Kaligineedi and V. K. Bhargava, "Sensor allocation and quantization schemes for multi-band cognitive radio cooperative sensing system," IEEE Transactions on Wireless Communications, vol. 10, no. 1, pp. 284-293, Jan. 2011. Article (CrossRef Link).

[12] W. Zhang, R. K. Mallik, and K. B. Letaief, "Optimization of cooperative spectrum sensing with energy detection in cognitive radio networks," IEEE Transactions on Wireless Communications, vol. 8, no. 12, pp. 5761-5766, Dec. 2009. Article (CrossRef Link).

[13] W. Han, J. Li, Z. Tian, and Y. Zhang, "Efficient cooperative spectrum sensing with minimum overhead in cognitive radio," IEEE Transactions on Wireless Communications, vol. 9, no. 10, pp. 3006-3011, Oct. 2010. Article (CrossRef Link).

[14] J. Lai, E. Dutkiewicz, R. P. Liu, and R. Vesilo, "Performance optimization of cooperative spectrum sensing in cognitive radio networks," in Proc. of IEEE Wireless Communications and Networking Conference (WCNC), Apr. 2013, pp. 631-636. Article (CrossRef Link).

[15] N. Han and H. Li, "Cooperative spectrum sensing with location information," IEEE Transactions on Vehicular Technology, vol. 61, no. 7, pp. 3015-3024, Sep. 2012. Article (CrossRef Link).

[16] W. Kadhim Saad, M. Ismaila , R. Nordina, and A. A. El-Saleh, "On the performance of cooperative spectrum sensing of cognitive radio networks in AWGN and Rayleigh fading environments," KSII Transactions on Internet and Information Systems, vol. 7, no. 8, pp. 1754-1769, Aug. 2013. Article (CrossRef Link).

[17] S. Sanayei and A. Nosratinia, "Opportunistic downlink transmission with limited feedback," IEEE Transactions on Information Theory, vol. 53, no. 11, pp. 4363-4372, Nov. 2007. Article (CrossRef Link).

[18] T. Yoo, N. Jindal, and A. Goldsmith, "Multi-antenna downlink channels with limited feedback and user selection," IEEE Journal on Selected Areas in Communications, vol. 25, no. 7, pp. 1478-1491, Sep. 2007. Article (CrossRef Link).

[19] D. Gesbert and M.-S. Alouini, "How much feedback is multi-user diversity really worth?" in Proc. of IEEE International Conference on Communications (ICC), pp. 234-238, Jun. 2004. Article (CrossRef Link). 
[20] J. So and J. M. Cioffi, “Multiuser diversity in a MIMO system with opportunistic feedback,” IEEE Transactions on Vehicular Technology, vol. 58, no. 9, pp. 4909-4918, Nov. 2009. Article (CrossRef Link).

[21] S. Atapattu, C. Tellambura, and H. Jiang, "Energy detection based cooperative spectrum sensing in cognitive radio networks," IEEE Transactions on Wireless Communications, vol. 10, no. 4, pp. 1232-1241, Sep. 2010. Article (CrossRef Link).

[22] J. Ma and Y. G. Li, "Soft combination and detection for cooperative spectrum sensing in cognitive radio networks,” in Proc. of IEEE Global Communications Conference (GLOBECOM), Nov. 2007, pp. 3139-3143. Article (CrossRef Link).

[23] Y.-C. Liang, Y. Zeng, E. C.Y. Peh, and A. T. Hoang, "Sensing-throughput tradeoff for cognitive radio networks,” IEEE Trans. Wireless Commun., vol. 7, no. 4, pp. 1326-1337, Apr. 2008. Article (CrossRef Link).

[24] H. Li, Q. Guo, and D. Huang, “Throughput analysis of opportunistic feedback for downlink multiuser diversity with capture effect,” IEEE Commun. Lett., vol. 16, no. 1, pp. 44-46, Jan. 2012. Article (CrossRef Link).

[25] S. Boyd and L. Vandenberghe, “Convex Optimization”, Cambridge, MA: Cambridge University Press, 2004. Article (CrossRef Link).

Jaewoo So received the B.S. degree in electronic engineering from Yonsei University, Seoul, Korea, in 1997, and received the M.S. and Ph.D. degrees in electrical engineering from the Korea Advanced Institute of Science and Technology (KAIST), Daejeon, Korea, in 1999 and 2002, respectively.

From 2001 to 2005, he was with IP One, Seoul, Korea, where he led several research projects and developed IEEE 802.11a/b/g products and heterogeneous network solutions. From 2005 to 2007, he was a Senior Engineer at Samsung Electronics, Suwon, Korea, where he involved in the design, performance evaluation, and development of mobile WiMAX systems and B3G wireless systems. From 2007 to 2008, he was a Postdoctoral Fellow in the Department of Electrical Engineering, Stanford University, Stanford, CA, USA. Since September 2008, he has been with the Department of Electronic Engineering, Sogang University, Seoul, Korea, where he is currently an Associate Professor. His current research interests include radio resource management, multiple antenna systems, cognitive radio networks, and IoT networks. He is a Senior Member of IEEE, a Life Member of KICS, a Member of IEEK, a Member of KSII, and a Member of IEICE. 